\title{
Safety Profile of the Adjuvanted Recombinant Zoster Vaccine in Immunocompromised Populations: An Overview of Six Trials
}

\author{
Marta López-Fauqued ${ }^{1} \cdot$ Maribel Co-van der Mee ${ }^{1} \cdot$ Adriana Bastidas $^{1,4} \cdot$ Pierre Beukelaers $^{1} \cdot$ Alemnew F. Dagnew $^{2,5}$. \\ Juan Jose Fernandez Garcia ${ }^{3} \cdot$ Anne Schuind ${ }^{2,6} \cdot$ Fernanda Tavares-da-Silva ${ }^{1}[0$
}

Accepted: 23 April 2021 / Published online: 11 June 2021

(c) The Author(s) 2021

\begin{abstract}
Introduction The adjuvanted recombinant zoster vaccine (RZV) has demonstrated high efficacy against herpes zoster in older adults and immunocompromised populations. We present comprehensive safety data from six clinical trials in immunocompromised populations (autologous hematopoietic stem cell transplant and renal transplant recipients, patients with hematologic malignancies, patients with solid tumors, and human immunodeficiency virus-infected adults) who are at an increased risk of herpes zoster.

Methods In all trials, immunocompromised adults $\geq 18$ years of age were administered RZV or placebo. Safety was evaluated in the total vaccinated cohort. Solicited adverse events (AEs) were collected for 7 days and unsolicited AEs for 30 days after each dose. Serious AEs, fatal serious AEs, and potential immune-mediated diseases were collected from dose 1 until 12 months post-last dose or study end. Data were pooled for solicited AEs; unsolicited AEs, (fatal) serious AEs, and potential immune-mediated diseases were analyzed for each individual trial. All AEs were analyzed for sub-strata of adults $18-49$ years of age and $\geq 50$ years of age.

Results In total, 1587 (RZV) and 1529 (placebo) adults were included in the pooled total vaccinated cohort. Solicited AEs were more common after RZV than placebo, were generally more common in the younger age stratum, and were mostly mild to moderate and resolved within 3 days (median duration). Unsolicited AEs and serious AEs were in line with underlying diseases and therapies. Across studies, the percentage of adults reporting one or more unsolicited AE was comparable between RZV and placebo, irrespective of age stratum. The percentage of adults reporting one or more serious AE, fatal serious AE, or potential immune-mediated diseases was generally similar for RZV and placebo, irrespective of age stratum. Overall, no safety concerns were identified.

Conclusions Recombinant zoster vaccine has a clinically acceptable safety profile. With the previously published vaccine efficacy and immunogenicity results, these data support a favorable benefit-risk profile of RZV vaccination in immunocompromised populations who are at an increased risk of herpes zoster.
\end{abstract}

\section{Plain Language Summary}

Varicella zoster virus leads to chickenpox after primary infection and herpes zoster upon reactivation of the latent virus. Older adults and immunocompromised people, whose immune system is impaired because of the age-related decline in immunity and their underlying disease and/or treatment, respectively, are at an increased risk of herpes zoster and its complications. Recombinant zoster vaccine has been approved to prevent herpes zoster and its complications in adults aged $\geq 50$ years in over 30 countries. In Europe, the vaccine has recently received approval to expand its use in adults aged 18 years or older who are at an increased risk of herpes zoster. We present an overview of the safety data from six clinical trials in immunocompromised patients vaccinated with recombinant zoster vaccine. We found that solicited adverse events were more common after the vaccine than placebo but that these were mild to moderate in intensity. Furthermore, the frequency of unsolicited adverse events was similar between the vaccine and placebo, and most of the reported adverse events and severe adverse

Fernanda Tavares-da-Silva

fernanda.tavares@gsk.com

Extended author information available on the last page of the article 
events (e.g., infections or tumors) could be attributed to the pre-existent diseases and/or therapies. As such, no safety concern was identified following the review of the available clinical data. This overview, together with the published efficacy data in the prevention of herpes zoster and the vaccine immunogenicity, provides useful medical information and supports the use of the recombinant zoster vaccine in an immunocompromised population at an increased risk of herpes zoster.

\section{Key Points}

Safety of the recombinant zoster vaccine was evaluated in six clinical trials in immunocompromised adults $\geq 18$ years of age

Adverse event profiles were generally balanced between the recombinant zoster vaccine and placebo and were generally consistent with the underlying diseases and/or therapies

Recombinant zoster vaccine has a favorable benefit-risk profile in adults $\geq 18$ years of age with diverse immunocompromising conditions

\section{Introduction}

Herpes zoster (HZ) results from the symptomatic reactivation of latent varicella-zoster virus in the dorsal root or cranial nerve ganglia and usually presents as a vesicular rash with a dermatomal unilateral distribution [1]. In generally healthy adults, the incidence of $\mathrm{HZ}$ increases from under five cases per 1000 person-years in people under 50 years of age (YOA) to over ten cases per 1000 person-years in people over 70 YOA $[2,3]$.

A decline in varicella-zoster virus-specific cell-mediated immunity increases the risk of $\mathrm{HZ}[4,5]$. In older adults, this decline occurs as a consequence of the age-related decline in immunity [6], but cell-mediated immunity impairments may also result from disease or immunosuppressive treatment [7-9]. As a result, immunocompromised (IC) populations are at an increased risk of $\mathrm{HZ}[10,11]$. The risk of $\mathrm{HZ}$ is, for instance, substantially elevated after kidney $[12,13]$ and autologous hematopoietic stem cell transplantation (auto-HSCT) [14-17], in patients with hematologic malignancies (HM) or solid malignancies [18-20], and in human immunodeficiency virus (HIV)-positive populations, even after the introduction of antiretroviral therapy [21-24]. Moreover, the severity and duration of $\mathrm{HZ}$ tend to be greater in IC populations, and IC patients are at a greater risk for HZ-associated complications [25, 26], disease dissemination [27], and recurrent $\mathrm{HZ}$ episodes [19, 28].
Antiviral therapy, if started within $72 \mathrm{~h}$ after onset of the zoster rash, can reduce the duration of the $\mathrm{HZ}$ rash and the formation of new lesions, but has limited impact on reducing the incidence or duration of post-herpetic neuralgia [29, 30]. Antiviral prophylaxis is commonly administered to HSCT recipients to prevent $\mathrm{HZ}$, but efficacy depends on adherence to treatment [31-33]. Moreover, the duration of prophylaxis is not standardized, and the risk of $\mathrm{HZ}$ is high once prophylaxis has stopped [31, 32].

Vaccination may provide an effective prophylactic measure to reduce the disease burden due to HZ. The live zoster vaccine (Zostavax; Merck and Co., Inc., New Jersey, United States) is a one-dose vaccine licensed for use in older adults, but live vaccines are generally contraindicated in IC populations because of their potential virulence $[34,35]$. A fourdose heat-inactivated varicella-zoster vaccine (V212; Merck and Co., Inc., New Jersey, United States) has been evaluated in IC adults [36-38], but it is not currently licensed for use. The two-dose adjuvanted recombinant zoster vaccine (RZV; Shingrix; GSK, Brentford, United Kingdom) is licensed for use in adults $\geq 50$ YOA in many countries worldwide, and in Europe also for use in adults $\geq 18$ YOA who are at an increased risk of $\mathrm{HZ}$ [39]. Recombinant zoster vaccine demonstrated $>90 \%$ efficacy against $\mathrm{HZ}$ in adults $\geq 50$ YOA $[40,41]$ and $>68 \%$ efficacy in auto-HSCT recipients $\geq 18$ YOA [42]. Post hoc analyses suggested a vaccine efficacy of $87 \%$ in adults with HM [43].

We previously published a comprehensive overview showing that RZV has an acceptable clinical safety profile when administered to adults $\geq 50$ YOA [44]. Data from clinical trials in IC populations have previously been published [42, 43, 45-48], but safety results from these trials have not been presented with the same level of detail as in the general older adult population.

In this article, we therefore present an extensive review of safety data across the six studies that have been completed to date (three phase III studies, one phase II/III study, and two supportive phase I/II clinical trials) in patients with IC conditions, including auto-HSCT and renal transplant (RT) recipients, patients with HM, patients with solid tumors (ST), and HIV-infected adults. Owing mainly to the heterogeneity of the underlying diseases and therapies of the IC study populations in the development program, data are pooled for all studies only for solicited adverse events (AEs) and presented per individual study for unsolicited AEs and other safety data. The method of collection of solicited AEs was similar across the six IC studies, hence it was considered 
appropriate to conduct a post hoc pooling of the data from all the studies. For unsolicited AEs and other safety data, the differences between study populations are anticipated to affect the ability to interpret pooled analyses; therefore, none of the studies had pre-specified plans for data pooling. We expect these data will inform policy decisions, recommendations, guidelines, and clinical practice protocols regarding the use of RZV in IC patients, who are at an increased risk of HZ.

\section{Methods}

\subsection{Study Design and Participants}

All six trials were randomized, placebo-controlled, observerblinded clinical trials conducted in parallel groups of IC participants. Methodologic details regarding the study design and objectives are presented in the primary publications, but a summary of the information relevant for this article is presented in Table 1. Anonymized individual participant data and study documents can be requested for further research from www.clinicalstudydatarequest.com.

\subsection{Study Vaccines}

Recombinant zoster vaccine contains $50 \mu \mathrm{g}$ of lyophilized recombinant glycoprotein $\mathrm{E}$ antigen and is reconstituted in the GSK proprietary AS01 ${ }_{\mathrm{B}}$ Adjuvant System (containing $50 \mu \mathrm{g}$ of 3-O-desacyl-4'-monophosphoryl lipid, $50 \mu \mathrm{g}$ of Quillaja saponaria Molina, fraction 21 [QS21, licensed by GSK from Antigenics LLC, a wholly owned subsidiary of Agenus Inc., a Delaware, USA corporation] and liposome).

Vaccinations were administered by qualified study personnel not involved in further data collection. Investigators collecting study data were unaware of whether participants were assigned to receive RZV or placebo.

\subsection{Collection of Safety Data}

Methods for the collection of safety data were presented in the primary publications and are detailed here in the Electronic Supplementary Material (ESM). Figure S1 in the ESM presents the general characteristics for all safety data presented in this article.

The exact duration of safety follow-up times differed between studies. Safety follow-up started after administration of the first dose. In participants in the RT, HM, ST, phase I/II auto-HSCT (auto-HSCT[I/II]), and HIV studies, safety follow-up was approximately 12 months after the last vaccination. In the phase III auto-HSCT (auto-HSCT[III]) study, follow-up was driven by $\mathrm{HZ}$ case accrual and ranged from 12 months to 4 years after the last vaccine dose, with a median of 29 months.

Solicited AEs were collected via diary cards for 7 days after each vaccination. Unsolicited AEs were collected for 30 days after each vaccination. Serious AEs (SAEs) and potential immune-mediated diseases (pIMDs) were collected from the first vaccination dose until 12 months after the last vaccination. Fatal SAEs were collected from the first vaccination until study end.

\subsection{Statistical Analysis}

Safety analyses of unsolicited AEs, SAEs, fatal SAEs, and pIMDs were conducted on the total vaccinated cohorts of each trial, consisting of all study participants receiving at least one dose of RZV or placebo. Analysis of solicited AEs was performed on the pooled data set consisting of all six clinical trials (Fig. S1 in the ESM). The results for the analysis of solicited AEs overall per subject are presented in this article. Analyses were descriptive and were calculated as percentages with exact $95 \%$ confidence intervals (CIs) using both Medical Dictionary for Regulatory Activities $\left(\right.$ MedDRA ${ }^{\circledR}$ ) System Organ Class (SOC) and Preferred Term (PT). Analyses of SAEs, fatal SAEs, and pIMDs were performed for up to 30 days and for 12 months after the last vaccination dose, but only analyses for 12 months after the last dose are presented here.

Descriptive data were generated by age strata. We clustered participants between 18 and 49 YOA (18-49 YOA) and participants 50 YOA and older ( $\geq 50$ YOA). Because of the comparatively small sample sizes in the RZV groups in the auto-HSCT(I/II) and HIV studies, we were not able to generate meaningful data for age strata in these studies.

\section{Results}

\subsection{Study Populations}

Across six clinical trials, 1587 study participants who received at least one dose of RZV were evaluated and compared with 1529 participants who received at least one dose of placebo. Of all RZV recipients, 443 (27.9\%) were between 18 and 49 YOA at study initiation and 1144 (72.1\%) were $\geq 50$ YOA at study initiation. Of all placebo recipients, $419(27.4 \%)$ were in the 18-49 YOA stratum and 1110 (72.6\%) were in the $\geq 50$ YOA stratum.

Within each clinical trial, demographic characteristics were comparable between RZV and placebo recipients. Detailed demographic characteristics are provided in Table S1 in the ESM. 


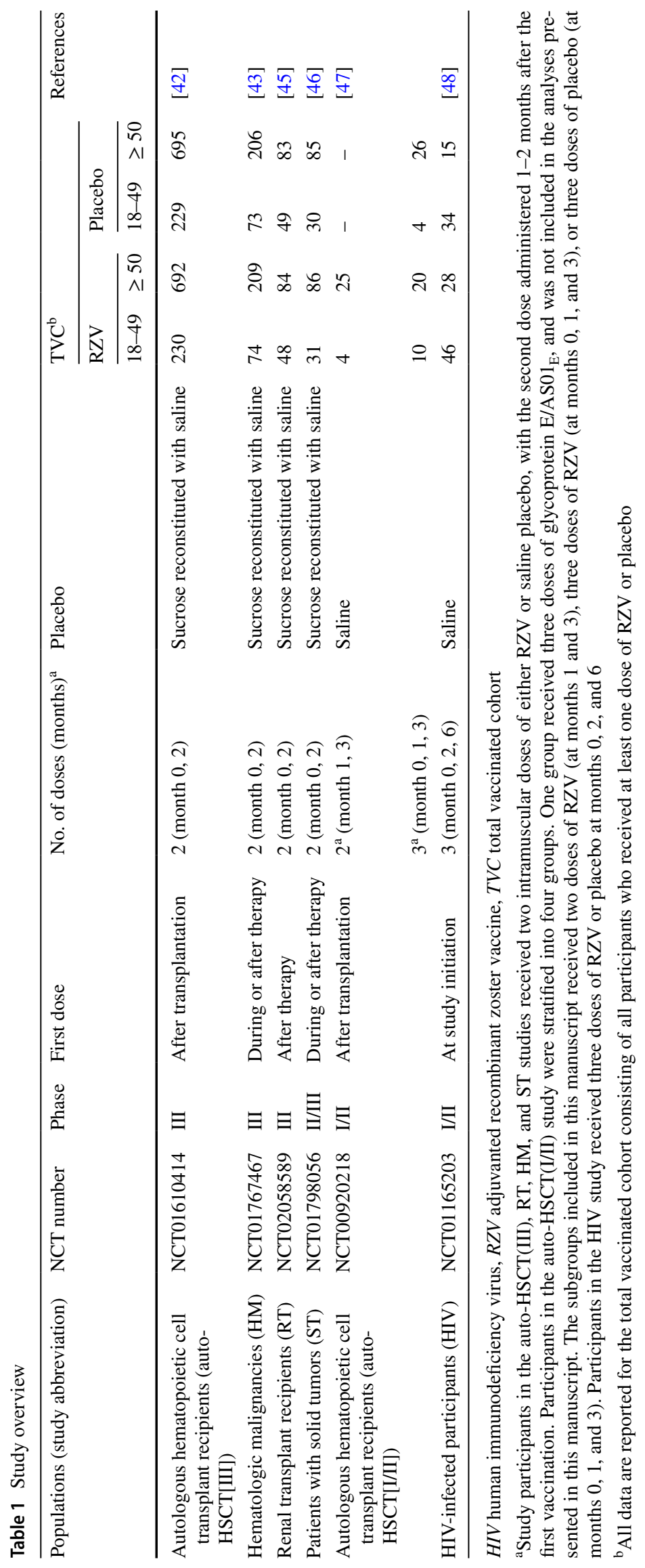




\subsection{Reactogenicity}

Recombinant zoster vaccine vaccination was associated with an increase in solicited local AEs compared with administration of placebo. The most frequently reported injection-site AE in both age strata was pain (Fig. S2 in the ESM). Most of the injection-site AEs were mild to moderate in intensity. In participants reporting injection-site AEs, the median duration of all AEs (pain, redness, and swelling) was 3 days in RZV recipients and varied from 1 to 2 days in placebo recipients. Grade 3 injection-site AEs were more common after administration of RZV compared with placebo (Fig. S2 in the ESM). Grade 3 redness and swelling were more common after dose 2 compared with dose 1 . In RZV recipients reporting grade 3 injection-site AEs, the median duration was 1 day for pain and redness, and 2 days for swelling. In placebo recipients, the only grade 3 injection-site $\mathrm{AE}$ reported was pain, with a median duration of 2 days. More RZV recipients 18-49 YOA appeared to report injection-site pain compared with participants $\geq 50$ YOA (Fig. S2 in the ESM). By sex, the incidence of redness and swelling at the injection site was higher in female than in male individuals.

Recombinant zoster vaccine vaccination was associated with an increase in solicited systemic AEs compared with administration of placebo (Fig. S3 in the ESM). Most solicited systemic AEs were mild to moderate in intensity. Most solicited systemic AEs were more common after dose 2 compared with dose 1 . However, symptoms after the second dose were mild to moderate and transient in nature, and the compliance with the second vaccine dose remained over 90 $\%$. Fatigue, myalgia, and headache were the most frequently reported solicited systemic AEs (Fig. S3 in the ESM). In participants reporting solicited systemic AEs, the median duration was generally comparable between RZV and placebo recipients, and varied from 1 to 3 days after RZV and from 1 to 5 days after placebo. Grade 3 solicited systemic AEs were more common after administration of RZV compared with placebo (Fig. S3 in the ESM). In RZV recipients reporting grade 3 solicited systemic AEs, the median duration was 1 day for all solicited systemic AEs except gastrointestinal symptoms, for which the median duration was 2 days. In placebo recipients, the median duration of grade 3 solicited systemic AEs varied from 1 to 3 days. More RZV recipients 18-49 YOA appeared to report solicited systemic AEs compared with RZV recipients $\geq 50$ YOA (Fig. $\mathrm{S} 3$ in the ESM). This difference in solicited systemic AEs was apparent for all AEs except gastrointestinal symptoms, which were comparable between the age cohorts. By sex, the incidence of solicited systemic AEs was generally higher in female than in male individuals.

\subsection{Unsolicited AEs}

In all studies, the percentage of study participants reporting any unsolicited symptoms was comparable between RZV and placebo recipients (Fig. 1). The percentage of study participants reporting unsolicited AEs within any SOC did not differ between RZV and placebo recipients in any of the clinical trials. The only exception was "neoplasm benign, malignant, and unspecified," reported by $2.1 \%$ of RZV and $7.9 \%$ of placebo recipients in the HM study (risk ratio: 0.27 [95\% CI 0.09-0.68]; $p=0.0033$ ).

In the auto-HSCT(III), RT, HM, and HIV studies, the most frequently reported unsolicited AEs per MedDRA SOC in RZV and placebo groups were "infections and infestations," "general disorders and administration site
Fig. 1 Unsolicited adverse events (AEs). auto-HSCT(I/II) autologous hematopoietic stem cell transplant recipients (phase I/II), auto-HSCT(III) autologous hematopoietic stem cell transplant recipients (phase III), $H I V$ human immunodeficiency virus-infected adults, $H M$ hematologic malignancies patients, $R T$ renal transplant recipients, $R Z V$ adjuvanted recombinant zoster vaccine, $S T$ solid tumors patients

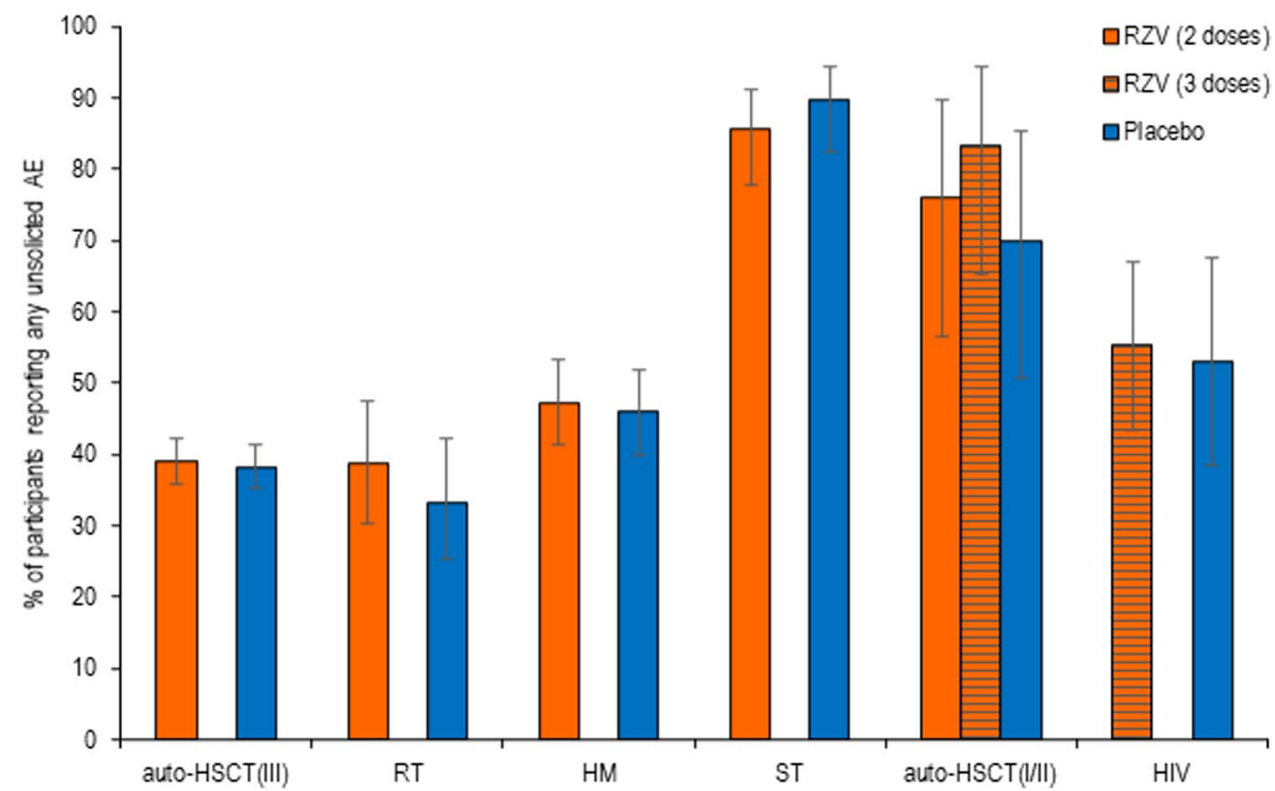

$\triangle$ Adis 
conditions," and "gastrointestinal disorders". In the ST study, "general disorders and administration site conditions," "gastrointestinal disorders," and "skin and subcutaneous tissue disorders" were most frequently reported. In the autoHSCT(I/II) study, "skin and subcutaneous tissue disorders," "respiratory, thoracic and mediastinal disorders," and "investigations" were most frequently reported (Table S2 in the ESM).

By MedDRA PT, most unsolicited AEs were reported by RZV and placebo recipients with comparable frequency (Table S2 in the ESM). The only exceptions were "upper respiratory tract infections", reported by $3.2 \%$ of RZV and $0.4 \%$ of placebo recipients in the HM study (risk ratio: 8.87 [95\% CI 1.23-388.9]; $p=0.0228$ ) and "acute myeloid leukemia", reported by $0.4 \%$ of RZV and $2.9 \%$ of placebo recipients in the HM study (risk ratio: 0.12 [95\% CI 0.00-0.92]; $p=$ $0.0371)$.

In the studies in which age-associated differences were assessed, the percentage of RZV recipients reporting any unsolicited symptoms was comparable between participants
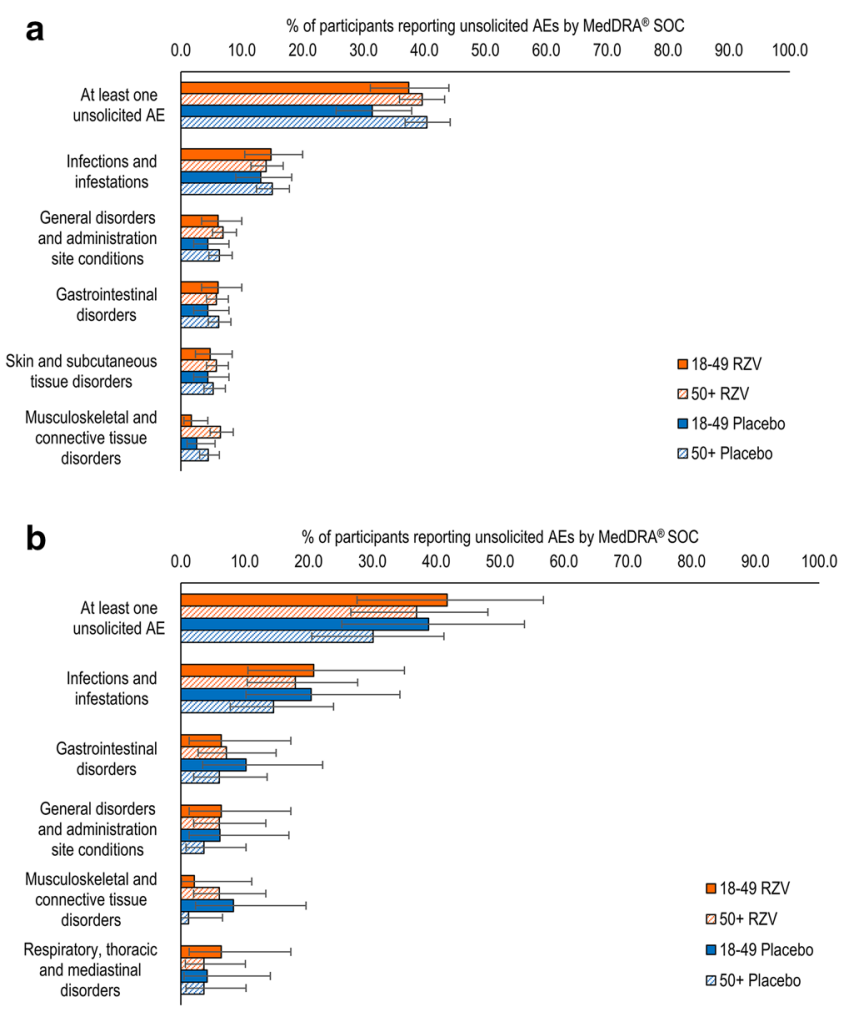

Fig. 2 Unsolicited adverse events (AEs) by age. a Adjuvanted recombinant zoster vaccine (RZV) and placebo in autologous hematopoietic cell transplant recipients [phase III] (Bastidas et al. [42]); b RZV and placebo in renal transplant recipients (Vink et al. [45]); c RZV and placebo in patients with hematologic malignancies (Dagnew et al. [43]); and d RZV and placebo in patients with solid organ tumors (Vink et al. [46]). Note: Figure panels present the percentage of RZV recipients reporting at least one unsolicited adverse event categorized
18-49 YOA and participants $\geq 50$ YOA (Fig. 2). By MedDRA SOC, most unsolicited AEs in RZV recipients were reported with comparable frequency by the populations $18-49$ YOA and $\geq 50$ YOA. Among the five most frequently reported unsolicited AEs by SOC, "musculoskeletal and connective tissue disorders" was the only exception, which was more common in recipients $\geq 50$ YOA than 18-49 YOA in the auto-HSCT(III) study (Fig. 2). In the $\geq 50$ YOA stratum, the percentage of participants reporting any unsolicited AE within the SOC of "musculoskeletal and connective tissue disorders" in the auto-HSCT(III) study was comparable between RZV and placebo recipients $(6.5 \%$ [95\% CI 4.8-8.6] and 4.5\% [95\% CI 3.1-6.3], respectively), as in all studies.

\subsection{SAEs}

The percentage of study participants reporting any SAE, including fatal SAEs, from the first dose until 12 months after receiving the last vaccination, was comparable between

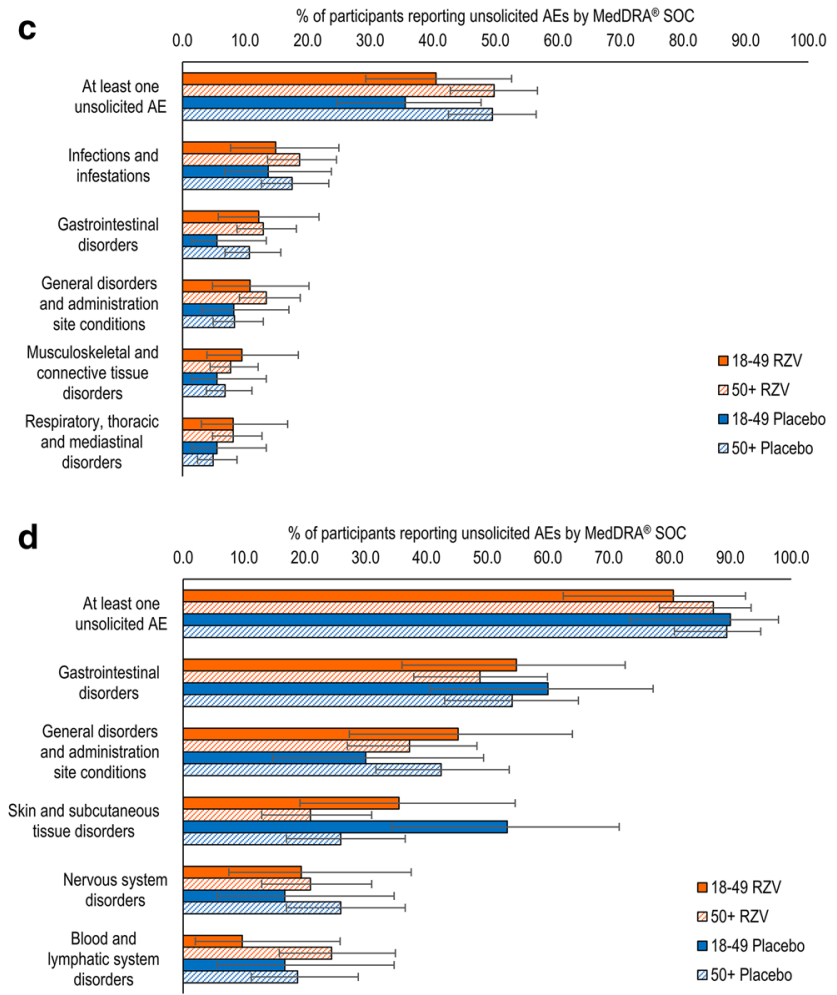

under that Medical Dictionary for Regulatory Activities (MedDRA) System Organ Class (SOC). Only the five SOCs with the greatest percentage of RZV recipients reporting any event are presented here. Because of the comparatively small sample sizes in the RZV groups in the studies with autologous hematopoietic cell transplant recipients (phase I/II) and human immunodeficiency virus-infected adults, we were not able to generate meaningful data for age strata in these studies 
RZV and placebo recipients in all six studies (Fig. 3 and Table S3 in the ESM). The percentage of study participants reporting SAEs within any single SOC did not differ between RZV and placebo recipients in any of the clinical trials, with the exception of "gastrointestinal disorders," which were reported more frequently in placebo recipients in the HM study (Table S3 in the ESM).

In general, the most frequently reported SAEs in RZV and placebo recipients were within the SOC "infections and infestations". Reflecting underlying disease in study participants, "neoplasms benign, malignant, and unspecified" were frequently reported in the auto-HSCT(III), HM, ST, and auto-HSCT(I/II) trials. Reported SAEs within other SOCs varied greatly between trials (Table $\mathrm{S} 3$ in the ESM). No single SAE was reported substantially more frequently by either RZV or placebo recipients in any of the trials (Table S3 in the ESM). The occurrence of an SAE was the most common reason for withdrawal from the study or treatment (in 9.6\% of study participants), participant withdrawal was balanced between the RZV and placebo recipients, and $78.4 \%$ of vaccinated participants completed the trials.

Within each age cohort, the percentage of study participants reporting SAEs was comparable between RZV and placebo recipients in all trials (Fig. 4). Recombinant zoster vaccine recipients $\geq 50$ YOA generally reported more SAEs than those 18-49 YOA (Fig. 4). There were no apparent differences in the incidence of SAEs between RZV and placebo recipients within either age stratum, except, in the auto-HSCT(III) trial, Hodgkin's disease was reported more frequently in the 18-49 YOA stratum (3.9\% [95\% CI 1.8-7.3]) than the $\geq 50$ YOA stratum (0 [95\% CI 0.0-0.5]) and plasma cell myeloma was reported more frequently in the $\geq 50$ YOA stratum $(7.7 \%[95 \%$
CI 5.8-9.9]) than in the 18-49 YOA stratum (2.6\% [95\% CI 1.0-5.6]) of the RZV treatment arm. For these PTs, a similar trend was observed in the placebo group. Placebo recipients 18-49 YOA reported more frequently Hodgkin's disease (0.9\% [95\% CI $0.1-3.1])$ than those $\geq 50$ YOA (0 [95\% CI 0.0-0.5]) and placebo recipients $\geq 50$ YOA reported more frequently plasma myeloma $(5.2 \%$ [95\% CI $3.7-7.1])$ than those $18-49$ YOA (2.2\% [95\% CI 0.7-5.0]).

\subsection{Fatal SAEs}

The percentage of study participants with a fatal SAE, from the first dose until 12 months after receiving the last vaccination, was comparable between RZV and placebo recipients in all studies (Fig. 5 and Table S4 in the ESM). Most fatal SAEs were categorized under "neoplasms benign, malignant, and unspecified" and "infections and infestations." There were no apparent differences between RZV and placebo recipients in fatal SAEs reported by SOC or PT.

In all studies, the percentage of RZV recipients with a fatal SAE was higher in participants $\geq 50$ YOA than in participants 18-49 YOA (Fig. 6). There were no differences in the incidence of any fatal SAEs between RZV and placebo recipients within either age stratum at the SOC and PT levels in any of the clinical trials, except for plasma cell myeloma in the auto-HSCT(III) study, which was reported more frequently in the $\geq 50$ YOA stratum (4.2\% [95\% CI 2.8-6.0]) than in the $18-49$ YOA stratum $(0.4 \%$ [95\% CI 0.0-2.4]) in the RZV group. Similarly, this PT was more frequently reported in placebo recipients $\geq 50$ YOA $(3.7 \%$ [95\% CI 2.5-5.4]) than in those $18-49$ YOA (1.3\% [95\% CI 0.3-3.8]).
Fig. 3 Serious adverse events (SAEs). auto-HSCT(I/II) autologous hematopoietic stem cell transplant recipients (phase I/II), auto-HSCT(III) autologous hematopoietic stem cell transplant recipients (phase III), $H M$ hematologic malignancies patients, HIV human immunodeficiency virus-infected adults, $R T$, renal transplant recipients, $R Z V$ adjuvanted recombinant zoster vaccine, $S T$ solid tumors patients

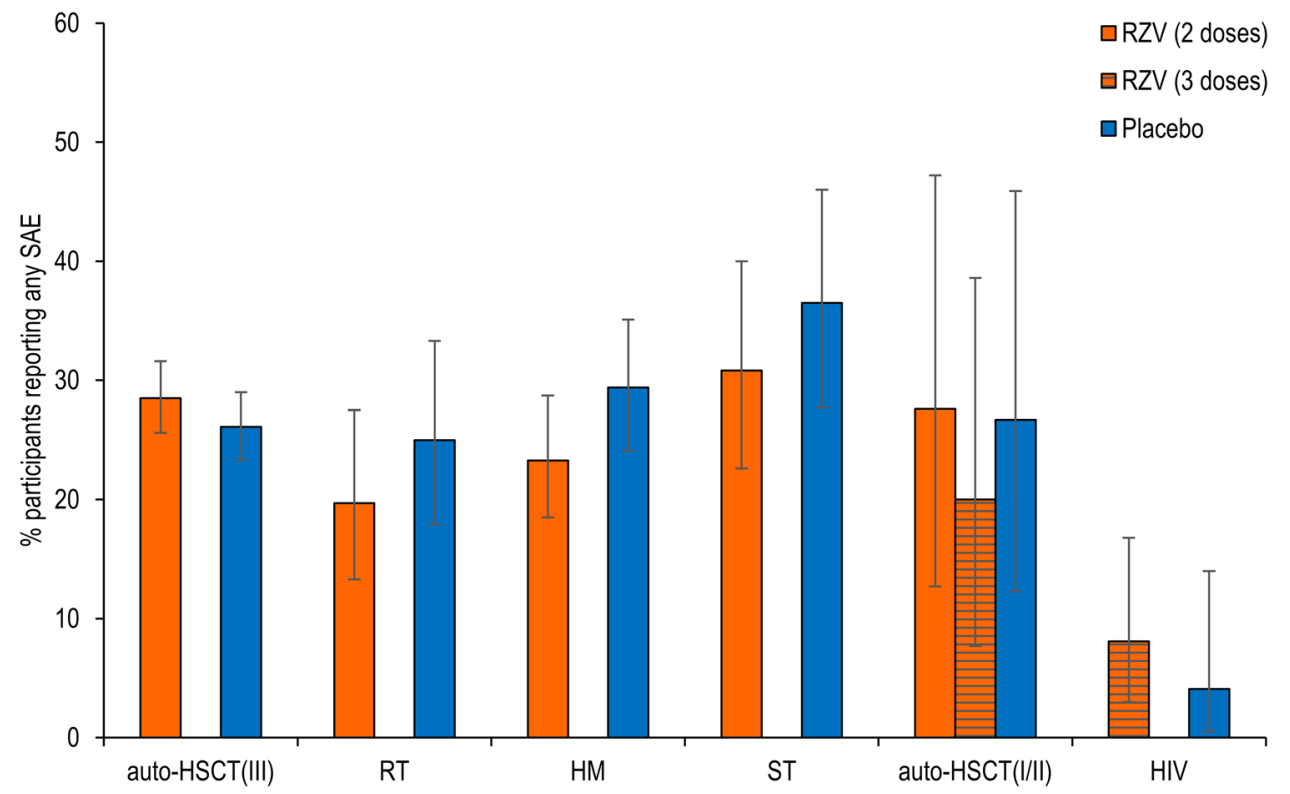

$\triangle$ Adis 


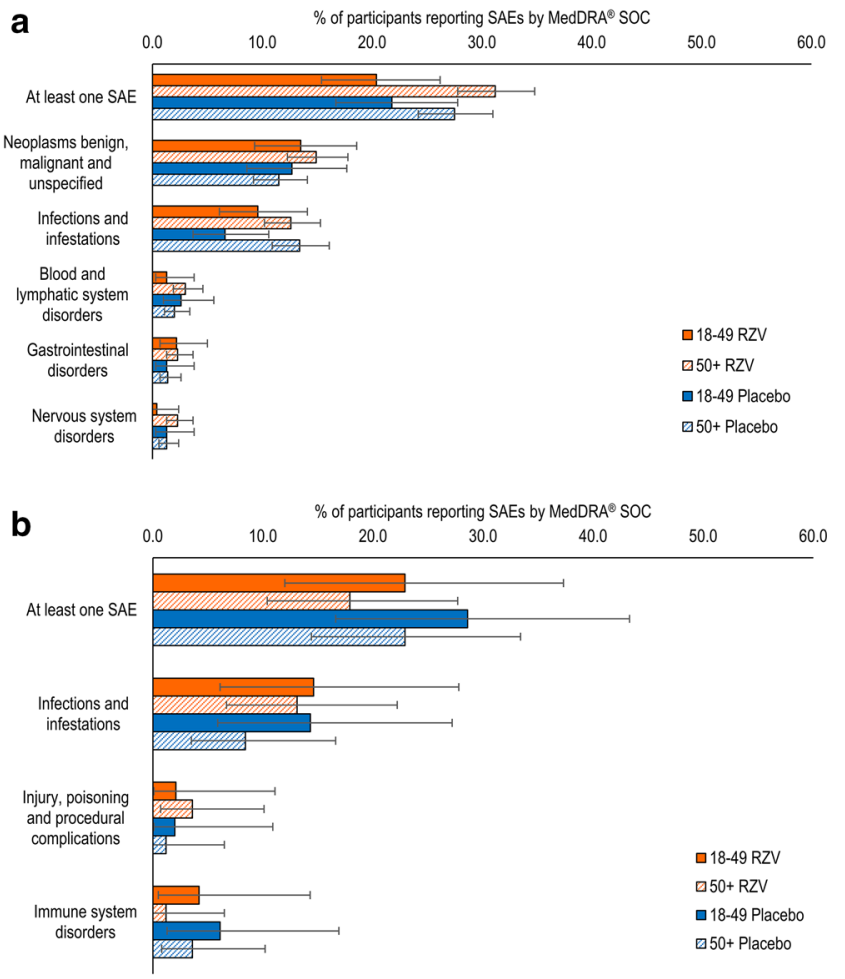

Fig. 4 Serious adverse events (SAEs) by age. a Adjuvanted recombinant zoster vaccine RZV and placebo in autologous hematopoietic cell transplant recipients [phase III] (Bastidas et al. [42]); b RZV and placebo in renal transplant recipients (Vink et al. [45]); c RZV and placebo in patients with hematologic malignancies (Dagnew et al. [43]); and d RZV and placebo in patients with solid organ tumors (Vink et al. [46]). Figure panels present the percentage of RZV recipients reporting at least one unsolicited adverse event categorized

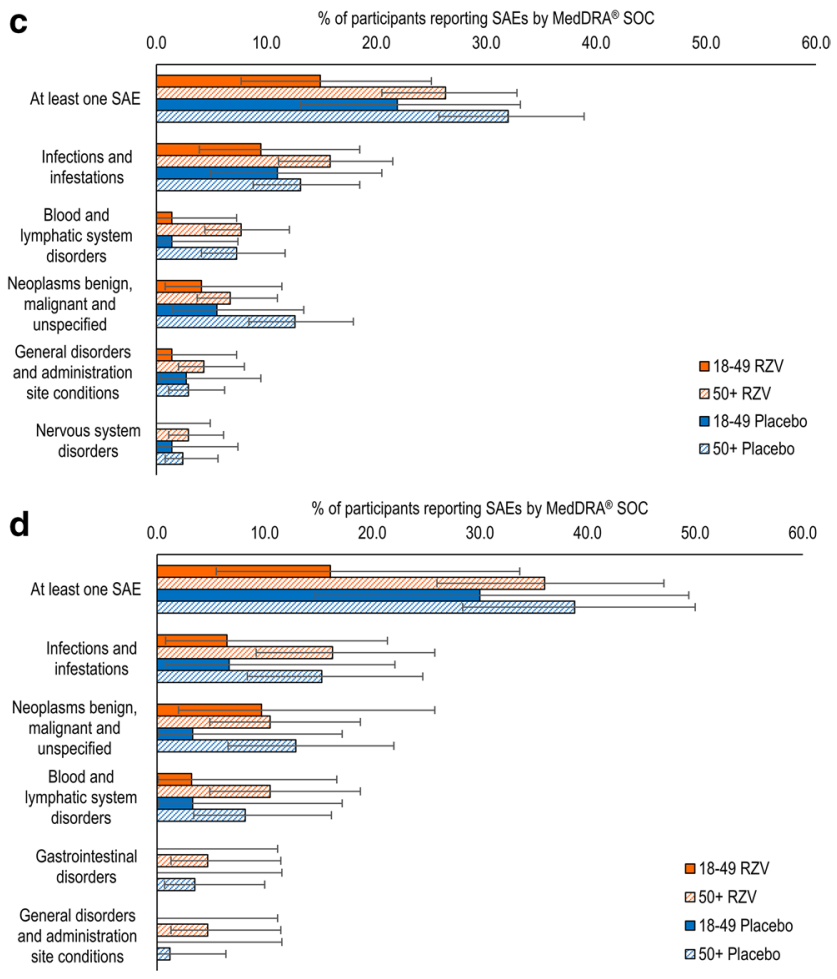

under that Medical Dictionary for Regulatory Activities (MedDRA) System Organ Class (SOC). Only the five SOCs with the greatest percentage of RZV participants reporting any event are presented here. Because of the comparatively small sample sizes in the RZV groups in the studies with autologous hematopoietic stem cell transplant recipients (phase I/II) and human immunodeficiency virus-infected adults, we were not able to generate meaningful data for age strata in these studies

\section{6 pIMDs}

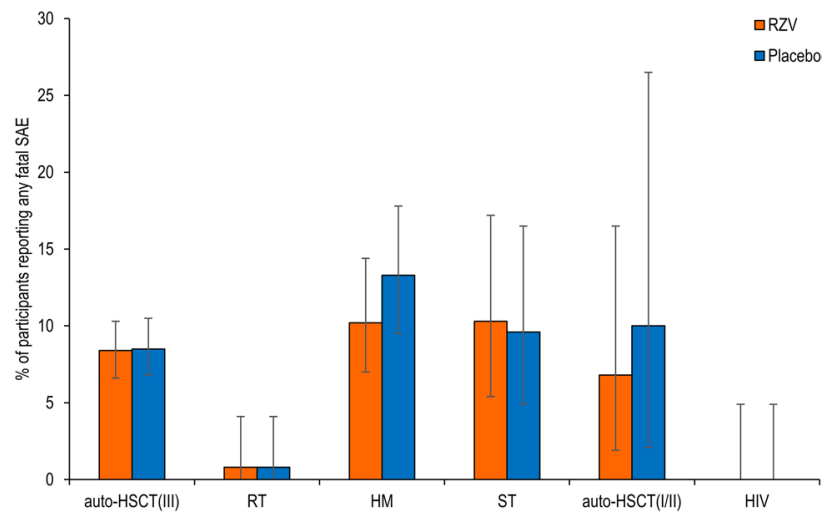

In all studies, the percentage of study participants reporting at least one pIMD was comparable between RZV and placebo recipients (Table $\mathrm{S} 5$ in the ESM). The most frequently reported pIMDs were not clustered within any specific SOC (Table S5 in the ESM).

\section{Discussion}

We presented a comprehensive overview of safety data from six clinical trials comparing RZV and placebo in patients who were immunosuppressed because of underlying diseases and/or therapy and included the following five distinct populations: auto-HSCT recipients, patients with HM (e.g., multiple myeloma, non-Hodgkin T-cell lymphoma, Hodgkin lymphoma), patients with ST (e.g., breast tumor, colorectal cancer, lung cancer), RT recipients, and patients with HIV. All studies in the IC program were placebo controlled and 


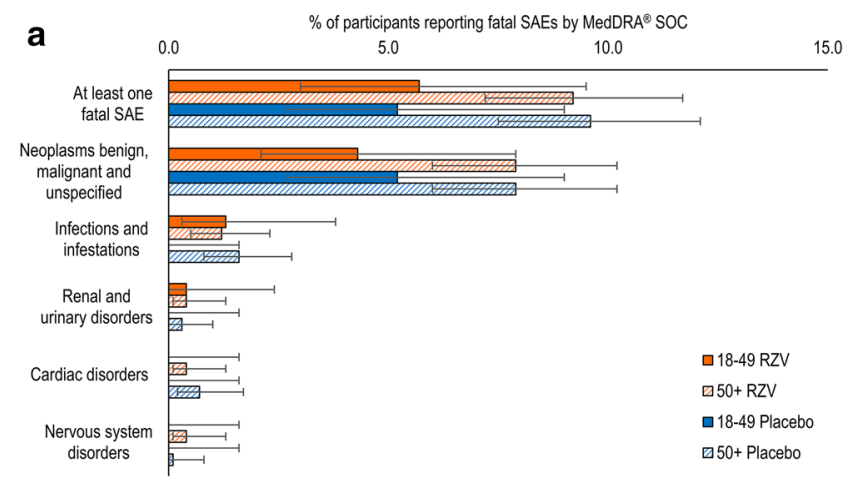

b

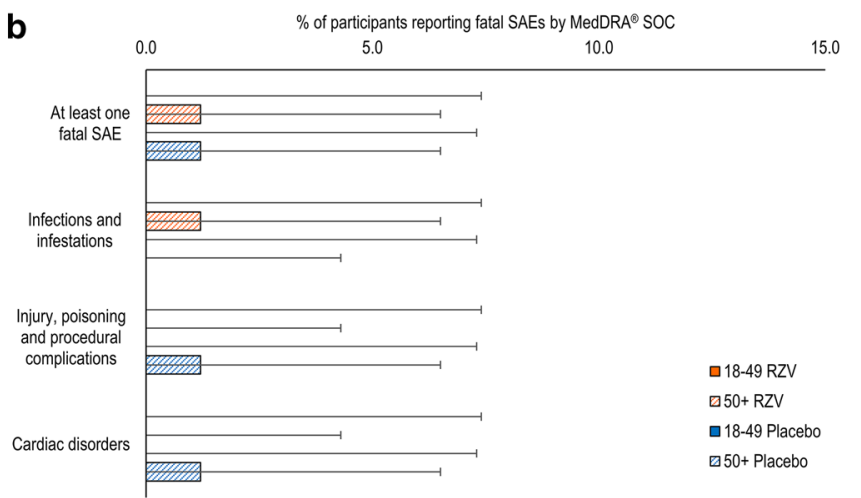

Fig. 6 Fatal serious adverse events (SAEs) by age. a Adjuvanted recombinant zoster vaccine (RZV) and placebo in autologous hematopoietic cell transplant recipients [phase III] (Bastidas et al. [42]); b RZV and placebo in renal transplant recipients (Vink et al. [45]); c RZV and placebo in patients with hematologic malignancies (Dagnew et al. [43]); and $\mathbf{d}$ RZV and placebo in patients with solid organ tumors (Vink et al. [46]). Figure panels present the percentage of RZV recipients reporting at least one unsolicited adverse event cat-

had similar design and methods for assessing many prespecified safety events.

Overall, no safety concern was identified in any of the populations studied. In all trials, the incidence of unsolicited AEs, SAEs, fatal SAEs, and pIMDs was generally balanced between RZV and placebo groups. There were, however, marked differences in the incidence of unsolicited AEs and SAEs between studies. We consider that these differences result from the heterogenicity of the underlying diseases and therapies of the IC study populations in the development program, the baseline status of the participants, as well as the timing of vaccinations in relation to immunosuppressive treatments. Most participants with ST were vaccinated before the initiation of chemotherapy (a small number were vaccinated at the start of and concurrently with a chemotherapeutic cycle; see primary publication for details [44]), the majority of patients with HM were vaccinated after the completion of the chemotherapy course, whereas participants in the auto-HSCT(I/II) and auto-HSCT(III) studies were undergoing post-transplant immune reconstruction,

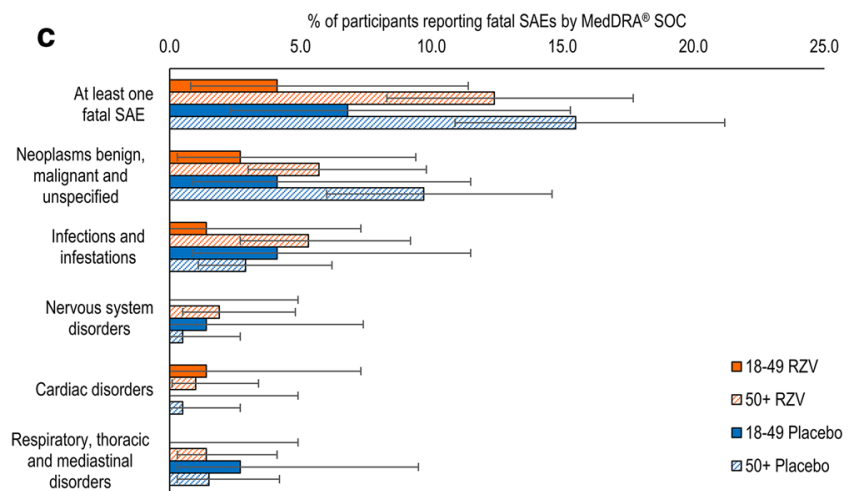

d

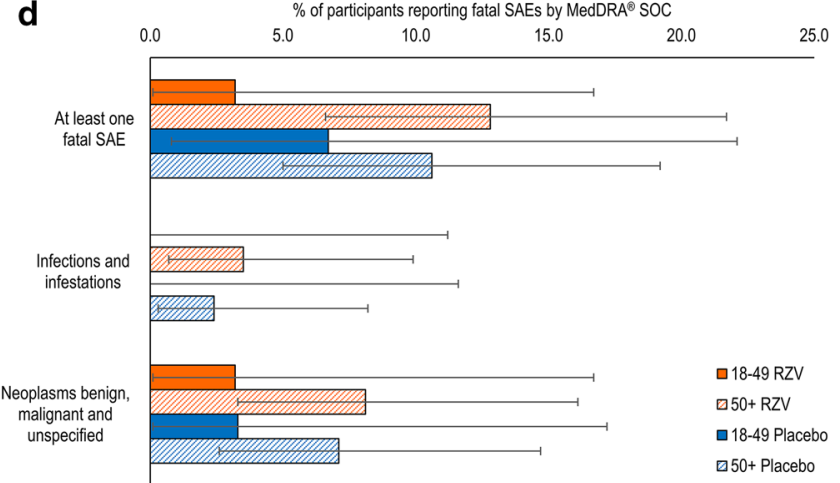

egorized under that Medical Dictionary for Regulatory Activities (MedDRA) System Organ Class (SOC). Only the five SOCs with the greatest percentage of RZV participants reporting any event are presented here. Because of the comparatively small sample sizes in the RZV groups in the studies with autologous hematopoietic cell transplant recipients (phase I/II) and human immunodeficiency virusinfected adults, we were not able to generate meaningful data for age strata in these studies

and patients receiving a RT were undergoing post-transplant immunosuppressive regimens when they were administered RZV.

Vaccine reactogenicity was analyzed for the pooled data from all IC studies. Recombinant zoster vaccine is associated with increased local and systemic AEs. The reactogenicity profile of RZV in the IC populations studied here did not differ markedly from that observed in healthy adults $\geq 50$ YOA [40, 41]. This suggests that underlying medical conditions and concomitant therapies do not negatively affect vaccine reactogenicity beyond what is seen in adults who are generally healthy. We note, however, that the percentage of placebo recipients reporting solicited systemic AEs was substantially greater in IC populations compared with the general population of older adults [44], which is likely owing to their underlying conditions and treatment that can also be associated with symptoms such as fatigue, headache, and gastrointestinal symptoms.

We observed a general trend toward a lower incidence of systemic AEs in the older age cohort compared with 
participants 18-49 YOA. In line with these findings, we previously reported that the number of study participants reporting solicited AEs after RZV vaccination decreased with age in studies in older adults [44]. These differences might be due to a higher tolerance to pain and illness symptoms gained with life experience and/or waning innate immune defense mechanisms, as supported by the observation that older people display lower systemic levels of interleukin-6, interleukin-10, and C-reactive protein after vaccination that could contribute to their tendency to report fewer systemic AEs $[49,50]$.

Overall, the incidence of unsolicited AEs was balanced between RZV and placebo recipients in all phase III IC trials. No unsolicited AEs per any MedDRA SOC were reported more frequently by RZV than placebo recipients, and this was true for both the overall population and for the populations in either age stratum. Therefore, we consider that the additional burden of RZV is limited in these IC populations that are already subject to a multitude of AEs associated with their underlying disease and medical therapies.

Overall, the majority of unsolicited AEs were infections and infestations, most commonly upper respiratory tract infections, which are common in the populations under study. The increase in "upper respiratory tract infection" (MedDRA PT) in RZV recipients was statistically significant in the HM study, but a difference was only seen in that trial. Similarly, "acute myeloid leukemia" (MedDRA PT) was observed significantly more often in placebo recipients in the HM study, with no difference observed in other trials. An additional analysis grouping upper respiratory tract infections with other PTs of the same medical context (highlevel term: upper respiratory tract infections) did not show evidence of an increased risk of these infections following vaccination with RZV (data not shown).

In general, there was no apparent difference in the incidence of unsolicited AEs between participants 18-49 YOA and those $\geq 50$ YOA, with the exception of "musculoskeletal and connective tissue disorders" in the auto-HSCT(III) efficacy study, which was among the five most frequently reported unsolicited AEs and occurred more commonly in older participants. Because we did not see these differences in the other studies, and the incidence was relatively low with an overlap in CIs, we consider that these were chance findings.

Our findings show that RZV vaccination is not associated with an increase in the incidence of SAEs and fatal SAEs, as these were balanced between RZV and placebo recipients. The incidence of SAEs and fatal SAEs was much greater in the $\geq$ 50 YOA age stratum in our study populations compared with previously studied populations of non-IC older adults [44], with the exception of fatal SAEs in the RT study, which only occurred in participants $\geq 50$ YOA. This increased number of
SAEs is due to underlying malignancies; for most of the fatal SAEs, although the time to occurrence was too long for any temporal association with vaccination, no imbalance between RZV and placebo recipients was observed. Most SAEs were reported under the MedDRA SOCs of "infections and infestations" and "neoplasms benign, malignant, and unspecified," in line with expectations for IC populations.

As for all adjuvanted vaccines, we carefully monitored the incidence of pIMDs following standardized disease-specific questionnaires [51]. Even though there are theoretic risks for pIMDs associated with adjuvanted vaccines, we have not previously seen any association between RZV vaccination and the onset or exacerbation of pIMDs in older adults [44]. The prevalence of pIMDs is rare and can vary depending on underlying conditions [52]. In the studies presented here, we did not find any indication of an imbalance in the incidence of both new onset and exacerbation of pIMDs between RZV and placebo recipients and no clusters in the nature of these AEs. However, the statistical power of clinical trials to detect very rare events is limited by the relatively small sample sizes, and we are therefore committed to continuing the evaluation of rare events such as pIMDs during post-licensure monitoring, where a larger population will be vaccinated.

The analyses present a number of limitations. The heterogeneity of the underlying diseases and treatment profiles may impact different aspects of the immune system in the patient populations presented in this article. In addition, the auto-HSCT(III) efficacy study contributed approximately $60 \%$ of the study participants. Finally, because of the fact that the conditions studied are more frequent with increasing age, over $70 \%$ of participants who received at least one dose of RZV in the IC clinical studies were 50 YOA or older.

Nonetheless, considering that the IC populations assessed are amongst the most medically vulnerable, we consider that the safety data generated are relevant to the $\geq 18$ YOA population at risk for $\mathrm{HZ}$ because of immunodeficiency or immunosuppression caused by disease and/or treatment. Moreover, because the immunosuppressive and immunomodulating treatments used in these populations are also widely used to treat other underlying conditions, such as autoimmune diseases and inflammatory conditions, we believe that the currently available data can be informative for the broad population of adults who is at increased risk of $\mathrm{HZ}$, including those patients with an immunodeficiency or immunosuppression caused by disease or therapy.

\section{Conclusions}

Together with the vaccine efficacy and immunogenicity data that were previously published, the safety results support a favorable benefit-risk profile of RZV vaccination in IC adults 18 YOA and older. 
Supplementary Information The online version contains supplementary material available at https://doi.org/10.1007/s40264-021-01076-w.

\section{Declaration}

Funding This study was sponsored and funded by GlaxoSmithKline Biologicals SA. GlaxoSmithKline Biologicals SA was involved in all stages of the conduct and analysis of the studies and covered the costs associated with the development and the publishing of the present article.

Conflicts of interest/Competing interests Maribel Co-van der Mee, Pierre Beukelaers, Juan Jose Fernandez Garcia, and Fernanda Tavaresda-Silva are employed by the GSK group of companies. Marta LópezFauqued, Juan Jose Fernandez Garcia, Adriana Bastidas, Alemnew F. Dagnew, and Anne Schuind were employees of the GSK group of companies during the design, initiation, conduct of the study and/or interpretation of the data. During the conduct of the study, Pierre Beukelaers, Juan Jose Fernandez Garcia, and Fernanda Tavares-da-Silva report personal fees from the GSK group of companies. Adriana Bastidas, Pierre Beukelaers, Alemnew F. Dagnew, Anne Schuind, and Fernanda Tavares-da-Silva hold shares in the GSK group of companies.

Ethics approval Not applicable.

Consent to participate Not applicable.

Consent for publication Not applicable.

Availability of data and material Not applicable.

Code availability Not applicable.

Authors' contributions MLF, AB, and FTS contributed to the concept and design of the study. MLF, MC, AD, and FTS contributed to data generation and collection. FTS, $\mathrm{PB}$, and $\mathrm{AD}$ contributed to the additional analysis of data. MLF, MC, AB, PB, AD, AS, JJFG, and FTS contributed to the analysis and interpretation of the data. All authors contributed to the writing/reviewing of the manuscript and approved the final version for submission. All authors vouch for the completeness and accuracy of all the data and the presented analyses.

Open Access This article is licensed under a Creative Commons Attribution-NonCommercial 4.0 International License, which permits any non-commercial use, sharing, adaptation, distribution and reproduction in any medium or format, as long as you give appropriate credit to the original author(s) and the source, provide a link to the Creative Commons licence, and indicate if changes were made. The images or other third party material in this article are included in the article's Creative Commons licence, unless indicated otherwise in a credit line to the material. If material is not included in the article's Creative Commons licence and your intended use is not permitted by statutory regulation or exceeds the permitted use, you will need to obtain permission directly from the copyright holder. To view a copy of this licence, visit http://creativecommons.org/licenses/by-nc/4.0/.

\section{References}

1. Cohen JI. Clinical practice: herpes zoster. N Engl J Med. 2013;369(3):255-63. https://doi.org/10.1056/NEJMcp1302674.
2. Kawai K, Gebremeskel BG, Acosta CJ. Systematic review of incidence and complications of herpes zoster: towards a global perspective. BMJ Open. 2014;4(6):e004833. https://doi.org/10. 1136/bmjopen-2014-004833.

3. Tseng HF, Bruxvoort K, Ackerson B, Luo Y, Tanenbaum H, Tian $\mathrm{Y}$, et al. The epidemiology of herpes zoster in immunocompetent, unvaccinated adults $\geq 50$ years old: incidence, complications, hospitalization, mortality, and recurrence. J Infect Dis. 2020;222:798-806. https://doi.org/10.1093/infdis/jiz652.

4. Gershon AA, Gershon MD, Breuer J, Levin MJ, Oaklander AL, Griffiths PD. Advances in the understanding of the pathogenesis and epidemiology of herpes zoster. J Clin Virol. 2010;48(Suppl. 1):S2-7. https://doi.org/10.1016/S1386-6532(10)70002-0.

5. Weinberg A, Zhang JH, Oxman MN, Johnson GR, Hayward AR, Caulfield MJ, et al. Varicella-zoster virus-specific immune responses to herpes zoster in elderly participants in a trial of a clinically effective zoster vaccine. J Infect Dis. 2009;200(7):1068 77. https://doi.org/10.1086/605611.

6. Ginaldi L, Loreto MF, Corsi MP, Modesti M, De Martinis M. Immunosenescence and infectious diseases. Microbes Infect. 2001;3(10):851-7. https://doi.org/10.1016/s1286-4579(01) 01443-5.

7. Meyers JD, Flournoy N, Thomas ED. Cell-mediated immunity to varicella-zoster virus after allogeneic marrow transplant. J Infect Dis. 1980;141(4):479-87. https://doi.org/10.1093/infdis/141.4. 479.

8. Cohen J, Straus S, Arvin A. Varicella-zoster virus and its replication, pathogenesis and management. In: Fields BN, Knipe DM, Howley PM, editors. Fields virology. 5th ed. Philadelphia: Wolters Kluwer Health/Lippincott Williams \& Wilkins; 2007.

9. Levin MJ, Bresnitz E, Popmihajlov Z, Weinberg A, Liaw KL, Willis $\mathrm{E}$, et al. Studies with herpes zoster vaccines in immune compromised patients. Expert Rev Vaccines. 2017;16(12):1217-30. https://doi.org/10.1080/14760584.2017.1395703.

10. Chen SY, Suaya JA, Li Q, Galindo CM, Misurski D, Burstin S, et al. Incidence of herpes zoster in patients with altered immune function. Infection. 2014;42(2):325-34. https://doi.org/10.1007/ s15010-013-0550-8.

11. Hata A, Kuniyoshi M, Ohkusa Y. Risk of Herpes zoster in patients with underlying diseases: a retrospective hospital-based cohort study. Infection. 2011;39(6):537-44. https://doi.org/10.1007/ s15010-011-0162-0.

12. Arness T, Pedersen R, Dierkhising R, Kremers W, Patel R. Varicella zoster virus-associated disease in adult kidney transplant recipients: incidence and risk-factor analysis. Transpl Infect Dis. 2008;10(4):260-8. https://doi.org/10.1111/j.1399-3062.2007. 00289.x.

13. Pergam SA, Forsberg CW, Boeckh MJ, Maynard C, Limaye AP, Wald A, et al. Herpes zoster incidence in a multicenter cohort of solid organ transplant recipients. Transpl Infect Dis. 2011;13(1):15-23. https://doi.org/10.1111/j.1399-3062.2010. 00547.x.

14. Locksley RM, Flournoy N, Sullivan KM, Meyers JD. Infection with varicella-zoster virus after marrow transplantation. J Infect Dis. 1985;152(6):1172-81. https://doi.org/10.1093/infdis/152.6. 1172.

15. Rogers JE, Cumpston A, Newton M, Craig M. Onset and complications of varicella zoster reactivation in the autologous hematopoietic cell transplant population. Transpl Infect Dis. 2011;13(5):480-4. https://doi.org/10.1111/j.1399-3062.2011. 00655.x.

16. Schuchter LM, Wingard JR, Piantadosi S, Burns WH, Santos GW, Saral R. Herpes zoster infection after autologous bone marrow transplantation. Blood. 1989;74(4):1424-7.

17. Sahoo F, Hill JA, Xie H, Leisenring W, Yi J, Goyal S, et al. Herpes zoster in autologous hematopoietic cell transplant recipients in 
the era of acyclovir or valacyclovir prophylaxis and novel treatment and maintenance therapies. Biol Blood Marrow Transplant. 2017;23(3):505-11. https://doi.org/10.1016/j.bbmt.2016.12.620.

18. Yenikomshian MA, Guignard AP, Haguinet F, LaCasce AS, Skarin AT, Trahey A, et al. The epidemiology of herpes zoster and its complications in Medicare cancer patients. BMC Infect Dis. 2015;15:106. https://doi.org/10.1186/s12879-015-0810-6.

19. Habel LA, Ray GT, Silverberg MJ, Horberg MA, Yawn BP, Castillo AL, et al. The epidemiology of herpes zoster in patients with newly diagnosed cancer. Cancer Epidemiol Biomark Prev. 2013;22(1):82-90. https://doi.org/10.1158/1055-9965. EPI-12-0815.

20. Rusthoven JJ, Ahlgren P, Elhakim T, Pinfold P, Stewart L, Feld R. Risk factors for varicella zoster disseminated infection among adult cancer patients with localized zoster. Cancer. 1988;62(8):1641-6. https://doi.org/10.1002/1097-0142(19881 015)62:8\%3c1641::aid-cncr2820620831\%3e3.0.co;2-d.

21. Blank LJ, Polydefkis MJ, Moore RD, Gebo KA. Herpes zoster among persons living with HIV in the current antiretroviral therapy era. J Acquir Immune Defic Syndr. 2012;61(2):203-7. https:// doi.org/10.1097/QAI.0b013e318266cd3c.

22. Jansen K, Haastert B, Michalik C, Guignard A, Esser S, Dupke $\mathrm{S}$, et al. Incidence and risk factors of herpes zoster among hivpositive patients in the german competence network for HIV/ AIDS (KompNet): a cohort study analysis. BMC Infect Dis. 2013;13:372. https://doi.org/10.1186/1471-2334-13-372.

23. Gebo KA, Kalyani R, Moore RD, Polydefkis MJ. The incidence of, risk factors for, and sequelae of herpes zoster among HIV patients in the highly active antiretroviral therapy era. J Acquir Immune Defic Syndr. 2005;40(2):169-74. https://doi.org/10.1097/ 01.qai.0000178408.62675.b0.

24. Feller L, Wood NH, Lemmer J. Herpes zoster infection as an immune reconstitution inflammatory syndrome in HIV-seropositive subjects: a review. Oral Surg Oral Med Oral Pathol Oral Radiol Endod. 2007;104(4):455-60. https://doi.org/10.1016/j.tripl eo.2007.02.001

25. Elliott KJ. Other neurological complications of herpes zoster and their management. Ann Neurol. 1994;35(Suppl.):S57-61. https:// doi.org/10.1002/ana.410350717.

26. Gnann JW Jr. Varicella-zoster virus: atypical presentations and unusual complications. J Infect Dis. 2002;186(Suppl. 1):S91-8. https://doi.org/10.1086/342963.

27. Rommelaere M, Marechal C, Yombi JC, Goffin E, Kanaan N. Disseminated varicella zoster virus infection in adult renal transplant recipients: outcome and risk factors. Transplant Proc. 2012;44(9):2814-7. https://doi.org/10.1016/j.transproceed.2012. 09.090 .

28. Yawn BP, Wollan PC, Kurland MJ, St Sauver JL, Saddier P. Herpes zoster recurrences more frequent than previously reported. Mayo Clin Proc. 2011;86(2):88-93. https://doi.org/10.4065/mcp. 2010.0618.

29. Bruxelle J, Pinchinat S. Effectiveness of antiviral treatment on acute phase of herpes zoster and development of post herpetic neuralgia: review of international publications. Med Mal Infect. 2012;42(2):53-8. https://doi.org/10.1016/j.medmal.2011.11.001.

30. Whitley RJ, Volpi A, McKendrick M, Wijck A, Oaklander AL. Management of herpes zoster and post-herpetic neuralgia now and in the future. J Clin Virol. 2010;48(Suppl. 1):S20-8. https://doi. org/10.1016/S1386-6532(10)70005-6.

31. Lee CJ, Savani BN, Ljungman P. Varicella zoster virus reactivation in adult survivors of hematopoietic cell transplantation: how do we best protect our patients? Biol Blood Marrow Transplant. 2018;24(9):1783-7. https://doi.org/10.1016/j.bbmt.2018.04.003.

32. Seo HM, Kim YS, Bang CH, Lee JH, Lee JY, Lee DG, et al. Antiviral prophylaxis for preventing herpes zoster in hematopoietic stem cell transplant recipients: a systematic review and meta-analysis. Antiviral Res. 2017;140:106-15. https://doi.org/ 10.1016/j.antiviral.2017.01.011.

33. Zhang D, Weiss T, Feng Y, Finelli L. Duration of antiviral prophylaxis and risk of herpes zoster among patients receiving autologous hematopoietic stem cell transplants: a retrospective, observational study. Adv Ther. 2017;34(7):1610-21. https://doi.org/10. 1007/s12325-017-0553-4.

34. European Medicines Agency. Zostavax: summary of product characteristics. https://www.ema.europa.eu/en/documents/productinformation/zostavax-epar-product-information_en.pdf. Accessed 10 Feb 2020.

35. Ljungman P. Vaccination of immunocompromised patients. Clin Microbiol Infect. 2012;18(Suppl. 5):93-9. https://doi.org/10. 1111/j.1469-0691.2012.03971.x.

36. Hata A, Asanuma H, Rinki M, Sharp M, Wong RM, Blume K, et al. Use of an inactivated varicella vaccine in recipients of hematopoietic-cell transplants. N Engl J Med. 2002;347(1):26-34. https://doi.org/10.1056/NEJMoa013441.

37. Mullane KM, Winston DJ, Wertheim MS, Betts RF, Poretz DM, Camacho LH, et al. Safety and immunogenicity of heat-treated zoster vaccine (ZVHT) in immunocompromised adults. J Infect Dis. 2013;208(9):1375-85. https://doi.org/10.1093/infdis/jit344.

38. Winston DJ, Mullane KM, Cornely OA, Boeckh MJ, Brown JW, Pergam SA, et al. Inactivated varicella zoster vaccine in autologous haemopoietic stem-cell transplant recipients: an international, multicentre, randomised, double-blind, placebo-controlled trial. Lancet. 2018;391(10135):2116-27. https://doi.org/10.1016/ S0140-6736(18)30631-7.

39. European Commission. Public health: union register of medicinal products. Shingrix product information. https://ec.europa.eu/ health/documents/community-register/html/h1272.htm. Accessed 23 Nov 2020.

40. Cunningham AL, Lal H, Kovac M, Chlibek R, Hwang SJ, DiezDomingo J, et al. Efficacy of the herpes zoster subunit vaccine in adults 70 years of age or older. N Engl J Med. 2016;375(11):101932. https://doi.org/10.1056/NEJMoa1603800.

41. Lal H, Cunningham AL, Godeaux O, Chlibek R, Diez-Domingo J, Hwang SJ, et al. Efficacy of an adjuvanted herpes zoster subunit vaccine in older adults. N Engl J Med. 2015;372(22):2087-96. https://doi.org/10.1056/NEJMoa1501184.

42. Bastidas A, de la Serna J, El Idrissi M, Oostvogels L, Quittet P, Lopez-Jimenez J, et al. Effect of recombinant zoster vaccine on incidence of herpes zoster after autologous stem cell transplantation: a randomized clinical trial. JAMA. 2019;322(2):123-33. https://doi.org/10.1001/jama.2019.9053.

43. Dagnew AF, Ilhan O, Lee WS, Woszczyk D, Kwak JY, Bowcock $\mathrm{S}$, et al. Immunogenicity and safety of the adjuvanted recombinant zoster vaccine in adults with haematological malignancies: a phase 3, randomised, clinical trial and post-hoc efficacy analysis. Lancet Infect Dis. 2019;19(9):988-1000. https://doi.org/10.1016/ S1473-3099(19)30163-X.

44. Lopez-Fauqued M, Campora L, Delannois F, El Idrissi M, Oostvogels L, De Looze FJ, et al. Safety profile of the adjuvanted recombinant zoster vaccine: pooled analysis of two large randomised phase 3 trials. Vaccine. 2019;37(18):2482-93. https://doi.org/10. 1016/j.vaccine.2019.03.043.

45. Vink P, Ramon Torrell JM, Sanchez Fructuoso A, Kim SJ, Kim SI, Zaltzman J, et al. Immunogenicity and safety of the adjuvanted recombinant zoster vaccine in chronically immunosuppressed adults following renal transplant: a phase 3 , randomized clinical trial. Clin Infect Dis. 2020;70(2):181-90. https://doi.org/10.1093/ $\mathrm{cid} / \mathrm{ciz} 177$.

46. Vink P, Delgado Mingorance I, Maximiano Alonso C, RubioViqueira B, Jung KH, Rodriguez Moreno JF, et al. Immunogenicity and safety of the adjuvanted recombinant zoster vaccine in patients with solid tumors, vaccinated before or during 
chemotherapy: a randomized trial. Cancer. 2019;125(8):1301-12. https://doi.org/10.1002/cncr.31909.

47. Stadtmauer EA, Sullivan KM, Marty FM, Dadwal SS, Papanicolaou GA, Shea TC, et al. A phase 1/2 study of an adjuvanted varicella-zoster virus subunit vaccine in autologous hematopoietic cell transplant recipients. Blood. 2014;124(19):2921-9. https:// doi.org/10.1182/blood-2014-04-573048.

48. Berkowitz EM, Moyle G, Stellbrink HJ, Schurmann D, Kegg S, Stoll M, et al. Safety and immunogenicity of an adjuvanted herpes zoster subunit candidate vaccine in HIV-infected adults: a phase 1/2a randomized, placebo-controlled study. J Infect Dis. 2015;211(8):1279-87. https://doi.org/10.1093/infdis/jiu606.

49. El Yousfi M, Mercier S, Breuillé D, Denis P, Papet I, Mirand PP, et al. The inflammatory response to vaccination is altered in the elderly. Mech Ageing Dev. 2005;126(8):874-81. https://doi.org/ 10.1016/j.mad.2005.03.008.

50. Hervé C, Laupèze B, Del Giudice G, Didierlaurent AM, Tavares Da Silva F. The how's and what's of vaccine reactogenicity. NPJ Vaccines. 2019;4:39. https://doi.org/10.1038/s41541-019-0132-6.

51. Tavares Da Silva F, De Keyser F, Lambert PH, Robinson WH, Westhovens R, Sindic C. Optimal approaches to data collection and analysis of potential immune mediated disorders in clinical trials of new vaccines. Vaccine. 2013;31(14):1870-6. https://doi. org/10.1016/j.vaccine.2013.01.042.

52. Abu-Shakra M, Buskila D, Ehrenfeld M, Conrad K, Shoenfeld Y. Cancer and autoimmunity: autoimmune and rheumatic features in patients with malignancies. Ann Rheum Dis. 2001;60(5):433-41. https://doi.org/10.1136/ard.60.5.433.

\title{
Authors and Affiliations
}

\section{Marta López-Fauqued ${ }^{1} \cdot$ Maribel Co-van der Mee ${ }^{1}$. Adriana Bastidas ${ }^{1,4}$. Pierre Beukelaers ${ }^{1} \cdot$ Alemnew F. Dagnew $^{2,5}$. Juan Jose Fernandez Garcia ${ }^{3} \cdot$ Anne Schuind ${ }^{2,6} \cdot$ Fernanda Tavares-da-Silva ${ }^{1}[0$}

\author{
Marta López-Fauqued \\ mlopezf82@gmail.com \\ Maribel Co-van der Mee \\ maribel.x.co@gsk.com \\ Adriana Bastidas \\ adrianabastidas.md@gmail.com \\ Pierre Beukelaers \\ pierre.x.beukelaers@gsk.com \\ Alemnew F. Dagnew \\ fitayehu@yahoo.com \\ Juan Jose Fernandez Garcia \\ jean_j_fernandez@outlook.com
}

Anne Schuind

aschuind@path.org

1 GSK, Avenue Fleming 20, 1300 Wavre, Belgium

2 GSK, Rockville, MD, USA

3 GSK, Rixensart, Belgium

4 Present Address: Current affiliation: Mithra Pharmaceuticals, Flemalle, Belgium

5 Present Address: Bill \& Melinda Gates Medical Research Institute, Cambridge, MA, USA

6 Present Address: PATH, Washington DC, WA, USA 